\title{
EXPANSION OF A CLASS OF FUNCTIONS INTO AN INTEGRAL INVOLVING ASSOCIATED LEGENDRE FUNCTIONS
}

\author{
NANIGOPAL MANDAL \\ Calcutta Mathematical Society \\ AE-374, Sector I \\ Salt Lake City \\ Calcutta-700 064, India
}

\author{
and $\quad$ B.N. MANDAL \\ Physical and Earth Science Division \\ Indian Statistical Institute \\ 203, B.T. Road \\ Calcutta-700 035, India
}

(Received February 4, 1992 and in revised form March 18, 1993)

\begin{abstract}
A theorem for expansion of a class of functions into an integral involving associated Legendre functions is obtained in this paper. This is a somewhat general integral expansion formula for a function $f(x)$ defined in $\left(x_{1}, x_{2}\right)$ where $-1<x_{1}<x_{2}<1$, which is perhaps useful in solving certain boundary value problems of mathematical physics and of elasticity involving conical boundaries.
\end{abstract}

KEY WORDS AND PHRASES. Integral expansion of a function, associated Legendre function, Mehler-Fok integral transform.

1991 AMS SUBJECT CLASSIFICATION CODE. 44.

\section{INTRODUCTION.}

Integral transforms are often used to solve the problems of mathematical physics involving linear partial differential equations and also other problems. Integral expansions involving spherical functions of a class of functions are known as Mehler-Fok type transforms. In these transform formulae, the subscript of the Legendre functions appear as the integration variable while its superscript is either zero or a fixed integer (see Sneddon [10]). There is another class of integral transforms involving associated Legendre functions somewhat related to the Mehler-Fok transforms, in which the superscript of the associated Legendre,function appears in the integration formula while the subscript (complex) is kept fixed. Felsen [2] first developed this type of transform formulae involving $P_{-1 / 2+i \tau}^{-\mu}(\cos \theta)$ as kernel where $0<\theta<\pi$ from a unique $\delta$ function representation. Later Mandal ([6], [7]) obtained somewhat similar types of two transform formulae from the solution of two appropriately designed boundary value problems. In the first type, the argument $x$ of $P_{-1 / 2+i \tau}^{-\mu}(x)$ ranges from -1 to 1 while in the second, the argument $z$ of $P_{-1 / 2+i \tau}^{\mu}(z)$ ranges from 1 to $\infty$. Recently Mandal and Guha Roy [8] used a similar technique to establish another Mehler-Fok type integral transform formula involving $P_{-1 / 2+i \tau}^{-\mu}(\cos \theta)$ as kernel $(0<\theta<\alpha)$.

In the present paper, an integral expansion of a class of functions defined in $\left(x_{1}, x_{2}\right)$ where $-1<x_{1}<x_{2}<1$, involving associated Legendre functions is obtained. Based on direct investigation of the properties of spherical functions, sufficient conditions which would establish the validity of this expansion formula for a wide class of functions are obtained in a manner 
similar to the ideas used in ([3]-[5]). The main result is given in section 2 in the form of a theorem. Recently, we have used a similar technique to establish another type of integral representation [9] involving $P_{-1 / 2+i \tau}^{-\mu}(\cosh \alpha)$ as kernel where $0<\alpha<\alpha_{0}$.

2. INTEGRAL EXPANSION OF A FUNCTION IN $\left(x_{1}, x_{2}\right)$ WHERE $-1<x_{1}<x_{2}<1$.

We present the main result of this paper in the form of the following theorem.

THEOREM. Let $f(x)$ be a given function defined on the interval $\left(x_{1}, x_{2}\right)$ where $-1<x_{1}<x_{2}<1$ and satisfies the following conditions:

(1) The function $f(x)$ is piecewise continuous and has a bounded variation in the open interval $\left(x_{1}, x_{2}\right)$.

(2) The function $f(x)\left(1-x^{2}\right)^{-1} \ln \left(1-x^{2}\right)^{-1} \in L\left(x_{1}, x_{2}\right),-1<x_{1}<x_{2}<1$.

Then we have

where

$$
\begin{aligned}
f(x)= & \sum_{k} \sigma_{k}\left\lceil( \frac { 1 } { 2 } + i \tau - i \sigma _ { k } ) \left\lceil\left(\frac{1}{2}-i \tau-i \sigma_{k}\right) \frac{M\left(x, x_{2} ; i \sigma_{k}\right)}{\left(\partial / \partial \sigma_{k}\right) M\left(x_{2}, x_{1} ; i \sigma_{\widehat{k}}\right)} F\left(\sigma_{\widehat{k}}\right)\right.\right. \\
& +\frac{1}{2 \pi i} \int_{-\infty}^{\infty} \sigma\left\lceil( \frac { 1 } { 2 } + i \tau - i \sigma ) \left\lceil\left(\frac{1}{2}-i \tau-i \sigma\right) \frac{M\left(x, x_{2} ; i \sigma\right)}{M\left(x_{2}, x_{1} ; i \sigma\right)} F(\sigma) d \sigma\right.\right.
\end{aligned}
$$

$$
\begin{gathered}
F(\sigma)=\int_{x_{1}}^{x_{2}} \frac{f(x)}{1-x^{2}} M\left(x, x_{1} ; i \sigma\right) d \sigma \\
-1<x_{1}<x_{2}<1, M(x, y ; i \sigma)=P_{-1 / 2+i \tau}^{i \sigma}(x) P_{-1 / 2+i \tau}^{i \sigma}(-y)-P_{-1 / 2+i \tau}^{i \sigma}(-x) P_{-1 / 2+i \tau}^{i \sigma}(y)
\end{gathered}
$$

and $\sigma_{k}{ }^{\prime} s, \sigma, \tau$ are real. The equation (2.2) may be regarded as an integral transform of the function $f(x)$ defined in $\left(x_{1}, x_{2}\right)$ and $(2.1)$ is its inverse. (2.1) and (2.2) together give the integral expansion of the function $f(x)$.

PROOF OF THE EXPANSION THEOREM. To prove this expansion theorem, we first note that the representation (cf. Erdélyi [1])

$$
P_{-1 / 2+i \tau}^{i \sigma}(x)=\left(\frac{1+x}{1-x}\right)^{i \sigma / 2} F\left(\frac{1}{2}+i \tau, \frac{1}{2}-i \tau ; 1-i \sigma ; \frac{1-x}{2}\right) /\lceil(1-i \sigma),
$$

$-1<x_{1}<x<x_{2}<1$, where $F(a, b ; c ; x)$ denotes the hypergeometric series, implies $P_{-1 / 2+i \tau}^{i \sigma}(x)$ is continuous in the region defined by $-1<x_{1}<x<x_{2}<1,-\infty<\sigma<\infty$ and satisfies the inequality

$$
\left|P_{-1 / 2+i \tau}^{i \sigma}(x)\right| \leq \sqrt{s h \pi \sigma / \pi \sigma} P_{-1 / 2+i \tau}(x),
$$

where the Legendre function $P_{-1 / 2+i \tau}(x)$ is positive.

Using (2.3) it follows from (2.2) that

$$
\begin{gathered}
\int_{x_{1}}^{x_{2}}\left|\frac{f(x)}{1-x^{2}}\left[P_{-1 / 2+i \tau}^{i \sigma}(x) P_{-1 / 2+i \tau}^{i \sigma}\left(-x_{1}\right)-P_{-1 / 2+i \tau}^{i \sigma}(-x) P_{-1 / 2+i \tau}^{i \sigma}\left(x_{1}\right)\right]\right| d x \\
\leq \sqrt{s h \pi \sigma / \pi \sigma} \int_{x_{1}}^{x_{2}} \frac{|f(x)|}{1-x^{2}}\left\{P_{-1 / 2+i \tau}(x) P_{-1 / 2+i \tau}\left(-x_{1}\right)-P_{-1 / 2+i \tau}(-x) P_{-1 / 2+i \tau}\left(x_{1}\right)\right\} d x,
\end{gathered}
$$

and this shows that the conditions imposed on $f(x)$ imply that the integral $F(\sigma)$ is absolutely and uniformly convergent for $\sigma \in[-T, T]$ where $T$ is a positive large number. Hence $F(\sigma)$ is continuous on $[-T, T]$ and the repeated integral

$$
J(x, T)=\frac{1}{2 \pi i} \int_{-T}^{T} \sigma\left(( \frac { 1 } { 2 } + i \tau - i \sigma ) \left\lceil\left(\frac{1}{2}-i \tau-i \sigma\right) \frac{M\left(x, x_{2} ; i \sigma\right)}{M\left(x_{2} ; x_{1} ; i \sigma\right)} d \sigma \cdot \int_{x_{1}}^{x_{2}} \frac{f(y)}{1-y^{2}} M\left(y, x_{1} ; i \sigma\right) d y\right.\right.
$$


is meaningful. Also, uniform convergence allows us to change the order of integration and write $J(x, T)$ as

where

$$
J(x, T)=\int_{x_{1}}^{x_{2}} \frac{f(y)}{1-y^{2}} K(x, y, T) d y .
$$

$$
K(x, y, T)=\frac{1}{2 \pi i} \int_{-T}^{T} \sigma\left\lceil\left(\frac{1}{2}+i \tau-i \sigma\right) \Gamma\left(\frac{1}{2}-i \tau-i \sigma\right) \frac{M\left(x, x_{2} ; \imath \sigma\right) M\left(y, x_{1} ; i \sigma\right)}{M\left(x_{2}, x_{1} ; i \sigma\right)} d \sigma .\right.
$$

Now we shall show that the kernel $K(x, y, T)$ is symmetric in the variables $x$ and $y$. By definition, we have

$$
\begin{gathered}
K(x, y, T)-K(y, x, T)=\frac{1}{2 \pi i} \int_{-T}^{T} \sigma \digamma\left(\frac{1}{2}+i \tau-i \sigma\right) \digamma\left(\frac{1}{2}-i \tau-i \sigma\right) \frac{1}{M\left(x_{2}, x_{1} ; i \sigma\right)} \\
\cdot\left[M\left(x, x_{2} ; i \sigma\right) M\left(y, x_{1} ; i \sigma\right)-M\left(y, x_{2} ; i \sigma\right) M\left(x_{1}, x_{1} ; i \sigma\right)\right] d \sigma .
\end{gathered}
$$

It follows from the properties of associated Legendre functions (cf. Erdélyi [1]) that the integrand in the above integral is an odd function of $\sigma$, hence the integral vanishes. Thus

$$
K(y, x, T)=K(x, y, T) .
$$

To investigate the behavior of $K(x, y, T)$ as $T \rightarrow \infty$, by writing $\mu=-i \sigma$, we write (2.5) as

$$
K(x, y, T)=\frac{1}{2 \pi i} \int_{-i T}^{i T} \mu\left\lceil\left(\frac{1}{2}+i \tau+\mu\right) \Gamma\left(\frac{1}{2}-i \tau+\mu\right) \frac{M\left(x, x_{2} ;-\mu\right) M\left(y, x_{1} ;-\mu\right)}{M\left(x_{2}, x_{1} ;-\mu\right)} d \mu .\right.
$$

Expression under the integral sign in (2.7) is analytic function fo the complex variable $\mu$ and it has no singularity in the semi-plane $R e \mu \geq 0$, except for simple poles at $\mu=-i \sigma_{k}$ ( $k$ is positive integer) (cf. Felsen [2]), where

$$
M\left(x_{2}, x_{1} ; i \sigma_{k}\right)=0, \sigma_{k}>0 .
$$

Completing the contour of integration on (2.7) with the $\operatorname{arc}\left\lceil_{T}\right.$ of radius $T$ situated in the semi-plane $R e \mu \geq 0$ and applying the residue theorem, we obtain

where

$$
K(x, y, T)=K_{1}(x, y, T)-\sum_{k} \sigma_{k}\left\lceil\left(\frac{1}{2}+i \tau-i \sigma_{k}\right) \Gamma\left(\frac{1}{2}-i \tau-i \sigma_{k}\right) \cdot \frac{M\left(x, x_{2} ; i \sigma_{k}\right) M\left(y, x_{1} ; i \sigma_{k}\right)}{\left(\partial / \partial \sigma_{k}\right) M\left(x_{2} ; x_{1} ; i \sigma_{k}\right),}\right.
$$

$$
K_{1}(x, y, T)=\frac{1}{2 \pi i} \int_{\Gamma_{T}} \mu\left\lceil\left(\frac{1}{2}+i \tau+\mu\right) \digamma\left(\frac{1}{2}-i \tau+\mu\right) \frac{M\left(x, x_{2} ;-\mu\right) \dot{M}\left(y, x_{1} ;-\mu\right)}{M\left(x_{2}, x_{1} ;-\mu\right)} d \mu .\right.
$$

Suppose that $y \leq x$. By virtue of the definition

$$
\begin{aligned}
& P_{-1 / 2+i \tau}^{-\mu}(x)=\left(\frac{1+x}{1-x}\right)^{-\mu / 2} \frac{1}{\lceil(1+\mu)}[1+0(|\mu|-1)] \\
& P_{-1 / 2+i \tau}^{-\mu}(-x)=\left(\frac{1-x}{1+x}\right)^{-\mu / 2} \frac{1}{\Gamma(1+\mu)}\left[1+0\left(|\mu|^{-1}\right)\right]
\end{aligned}
$$

Using (2.11) and asymptotic properties of the gamma function for large $\mu$, we conclude that

$$
\mu\left\lceil\left(\frac{1}{2}+i \tau+\mu\right) \Gamma\left(\frac{1}{2}-i \tau+\mu\right) \frac{M\left(x, x_{2} ;-\mu\right) M\left(y, x_{1} ;-\mu\right)}{M\left(x_{2}, x_{1} ;-\mu\right)}\right.
$$




$$
=\frac{\left[\left(\frac{1+x}{1-x} \frac{1-x_{2}}{1+x_{2}}\right)^{-\mu / 2}-\left(\frac{1-x}{1+x} \frac{1+x_{2}}{1-x_{2}}\right)^{-\mu / 2}\right]\left[\left(\frac{1+y}{1-y} \frac{1-x_{1}}{1+x_{1}}\right)^{-\mu / 2}-\left(\frac{1-y}{1+y} \frac{1+x_{1}}{1-x_{1}}\right)^{-\mu / 2}\right]}{\left[\left(\frac{1+x_{2}}{1-x_{2}} \frac{1-x_{1}}{1+x_{1}}\right)^{-\mu / 2}-\left(\frac{1-x_{2}}{1+x_{2}} \frac{1+x_{1}}{1-x_{1}}\right)^{-\mu / 2}\right]}
$$

Now introduce the new variables

$$
\xi=\frac{1}{2} \ln \frac{1+x}{1-x}, \eta=\frac{1}{2} \ln \frac{1+y}{1-y}, \alpha=\frac{1}{2} \ln \frac{1+x_{1}}{1-x_{1}} \text { and } \beta=\frac{1}{2} \ln \frac{1+x_{2}}{1-x_{2}} .
$$

Then, for large $\mu$, from (2.10) - (2.12) we obtain for $y \leq x$

$$
\begin{aligned}
K_{1}(x, y, T)= & \frac{1}{2 \pi i} \int_{\Gamma_{T}}[\exp \{-\mu(\xi-\eta)\}+\exp \{-\mu(2 \beta-2 a-\xi+\eta)\} \\
& -\exp \{-\mu(\xi+\eta-2 \alpha)\}-\exp \{-\mu(2 \beta-\xi-\eta)\}] d \mu \\
& \left.+O(1) \int_{O}^{\pi / 2} \exp \{-\mu(\xi-\eta) \cos \varphi\}+\exp \{-\mu(2 \beta-2 \alpha-\xi+\eta) \cos \varphi\}\right] \\
& -\exp \{-\mu(\xi+\eta-2 \alpha) \cos \varphi\}-\exp \{-\mu(2 \beta-\xi-\eta) \cos \varphi\}] d \varphi,
\end{aligned}
$$

Using the identity for $\alpha<\eta \leq \xi<\beta$.

we obtain for $y \leq x$,

$$
\frac{2}{\pi} \int_{0}^{\pi / 2} \exp \{-\lambda T \cos \varphi\} d \varphi \leq \frac{1-\exp (-\lambda T)}{\lambda T}, \lambda \geq 0,
$$

$$
\begin{aligned}
K_{1}(x, y, T)= & \frac{1}{\pi}\left[\frac{\sin T(\xi-\eta)}{\xi-\eta}+\frac{\sin T(2 \beta-2 \alpha-\xi+\eta)}{2 \beta-2 \alpha-\xi+\eta}-\frac{\sin T(\xi+\eta-2 a)}{\xi+\eta-2 a}\right. \\
& \left.-\frac{\sin T(2 \beta-\xi-\eta)}{2 \beta-\xi-\eta}\right]+O(1)\left[\frac{1-\exp \{-T(\xi-\eta)\}}{T(\xi-\eta)}+\frac{1-\exp \{-T(2 \beta-2 \alpha-\xi+\eta)\}}{T(2 \beta-2 \alpha-\xi+\eta)}\right. \\
& \left.-\frac{1-\exp \{-T(\xi+\eta-2 \alpha)\}}{T(\xi+\eta-2 \alpha)}-\frac{1-\exp \{-T(2 \beta-\xi-\eta)\}}{T(2 \beta-\xi-\eta)}\right], \quad \alpha<\eta \leq \xi<\beta,
\end{aligned}
$$

where the factor $O(1)$ is independent of $y$.

Again for $y \geq x$, we use the symmetry property (2.6) and the representation (2.10) of $K_{1}(x, y, T)$ with the variables $x, y$ replaced by $y, x$.

Now we write (2.4) as

$$
\begin{gathered}
J(x, T)=\int_{x_{1}}^{x} \frac{f(y)}{1-y^{2}} K_{1}(x, y, T) d y+\int_{x}^{x_{2}} \frac{f(y)}{1-y^{2}} K_{1}(x, y, T) d y \\
-\sum_{k} \sigma_{k} \Gamma\left(\frac{1}{2}+i \tau-i \sigma_{k}\right) \Gamma\left(\frac{1}{2}-i \tau-i \sigma_{k}\right) \frac{M\left(x, x_{2} ; i \sigma_{k}\right)}{\left(\partial / \partial \sigma_{k}\right) M\left(x_{2}, x_{1} ; i \sigma_{k}\right)} \int_{x_{1}}^{x_{2}} \frac{f(y)}{1-y^{2}} M\left(y, x_{1} ; i \sigma_{k}\right) d y \\
=J_{1}(x, T)+J_{2}(x, T)-\sum_{k} \sigma_{k}\left\lceil\left(\frac{1}{2}+i \tau-i \sigma_{k}\right) \Gamma\left(\frac{1}{2}-i \tau-i \sigma_{k}\right) \frac{M\left(x, x_{2} ; i \sigma_{k}\right)}{\left(\partial / \partial \sigma_{k}\right) M\left(x_{2}, x_{1} ; i \sigma_{k}\right)} \times\right. \\
\times \cdot \int_{x_{1}}^{x_{2}} \frac{f(y)}{1-y^{2}} M\left(y, x_{1} ; i \sigma_{k}\right) d y .
\end{gathered}
$$


Using (2.13) in $J_{1}$, we obtain

$$
\begin{aligned}
J_{1}(x, T)= & \frac{1}{\pi}\left[\int_{\alpha}^{\xi} f(\tanh \eta) \frac{\sin T(\xi-n)}{\xi-\eta} d \eta+\int_{\alpha}^{\xi} f(\tanh \eta) \frac{\sin T(2 \beta-2 \alpha-\xi+\eta)}{2 \beta-2 \alpha-\xi+\eta} d \eta\right. \\
-\int_{\alpha}^{\xi} f(\tanh \eta) & \left.\frac{\sin T(\xi+\eta-2 \alpha)}{\xi+\eta-2 \alpha} d \eta-\int_{\alpha}^{\xi} f(\tanh \eta) \frac{\sin T(2 \beta-\xi-\eta)}{2 \beta-\xi-\eta} d \eta\right] \\
& +O(1)\left[\int_{\alpha}^{\xi}|f(\tanh \eta)| \frac{1-\exp \{-T(\xi-\eta)\}}{T(\xi-\eta)} d \eta\right. \\
& +\int_{\alpha}^{\xi}|f(\tanh \eta)| \frac{1-\exp \{-T(2 \beta-2 \alpha-\xi+\eta)\}}{T(2 \beta-2 \alpha-\xi+\eta)} d \eta \\
& -\int_{\alpha}^{\xi}|f(\tanh \eta)| \frac{1-\exp \{-T(\xi+\eta-2 \alpha)\}}{T(\xi+\eta-2 \alpha)} d \eta \\
& \left.-\int_{\alpha}^{\xi}|f(\tanh \eta)| \frac{1-\exp \{-T(2 \beta-\xi-\eta)\}}{T(2 \beta-\xi-\eta)} d \eta\right]
\end{aligned}
$$

The conditions satisfied by $f(x)$ imply that $f(\tanh \eta) \in L(\alpha, \beta)$; hence, by virtue of Dirichlet's theorem, for $T \rightarrow \infty$

and

$$
\begin{gathered}
\frac{1}{\pi} \int_{\alpha}^{\xi} f(\tanh \eta) \frac{\sin T(\xi-\eta)}{\xi-\eta} d \eta=\frac{1}{2} f(\tanh \xi-o)+o(1) \\
=\frac{1}{2} f(x-o)+o(1) \\
\frac{1}{\pi} \int_{\alpha}^{\xi} f(\tanh \eta) \frac{\sin T(2 \beta-2 a-\xi+\eta)}{2 \beta-2 \alpha-\xi+\eta} d \eta=o(1) \\
\frac{1}{\pi} \int_{\alpha}^{\xi} f(\tanh \eta) \frac{\sin T(\xi+\eta-2 \alpha)}{\xi+\eta-2 a} d \eta=o(1)
\end{gathered}
$$

$$
\frac{1}{\hbar} \int_{\alpha}^{\xi} f(\tanh \eta) \frac{\sin T(2 \beta-\xi-\eta)}{2 \beta-\xi-\eta} d \eta=o(1) .
$$

Moreover, if the integral of integration is divided into the subintervals $(\xi-\delta, \xi)$ and $(\alpha, \xi-\delta)$ and if a sufficiently small positive $\delta$ (implying a sufficiently large $T$ ) is chosen, then we have

$$
\begin{gathered}
\int_{\alpha}^{\xi}|f(\tanh \eta)| \frac{1-\exp \{-T(\xi-\eta)\}}{T(\xi-\eta)} d \eta \\
\leq \frac{1}{\delta T} \int_{\alpha}^{\xi-\delta}|f(\tanh \eta)| d \eta+\int_{\xi-\delta}^{\xi}|f(\tanh \eta)| d \eta \\
=O\left(T^{-1}\right)+o(1)=o(1) \text { for } T \rightarrow \infty, \\
\xi \quad|f(\tanh \eta)| \frac{1-\exp \{-T(2 \beta-2 \alpha-\xi+\eta)\}}{T(2 \beta-2 \alpha-\xi+\eta)} d \eta \leq \frac{1}{\xi T} \int_{\alpha}^{\xi}|f(\tanh \eta)| d \eta
\end{gathered}
$$


and

$$
\begin{aligned}
& =O\left(T^{-1}\right)=o(1) \text { for } T \rightarrow \infty, \\
\int_{\alpha}^{\xi}|f(\tanh \eta)| \frac{1-\exp \{-T(\xi+\eta-2 \alpha)\}}{T(\xi+\eta-2 \alpha)} d \eta \leq & \frac{1}{\xi T} \int_{\alpha}^{\xi}|f(\tanh \eta)| d \eta \\
& =O\left(T^{-1}\right)=o(1) \text { for } T \rightarrow \infty,
\end{aligned}
$$

$$
\begin{aligned}
\int_{\alpha}^{\xi}|f(\tanh \eta)| \frac{1-\exp \{-T(2 \beta-\xi-\eta)\}}{T(2 \beta-\xi-\eta)} d \eta \leq & \frac{1}{\xi T} \int_{\alpha}^{\xi}|f(\tanh \eta)| d \eta \\
& =O\left(T^{-1}\right)=o(1) \text { for } T \rightarrow \infty .
\end{aligned}
$$

Thus (2.15) to (2.17) leads to

$$
\lim _{T \rightarrow \infty} J_{1}(\tanh \xi, T)=\frac{1}{2} f(\tanh \xi-o)=\frac{1}{2} f(x-o) .
$$

Similarly,

$$
\lim _{T \rightarrow \infty} J_{2}(\tanh \xi, T)=\frac{1}{2} f(\tanh \xi+o)=\frac{1}{2} f(x+o)
$$

Hence,

$$
\begin{array}{r}
\lim _{T \rightarrow \infty} J(x, T)=\frac{1}{2}[f(x+o)+f(x-o)]-\sum_{k} \sigma_{k}\left\lceil( \frac { 1 } { 2 } + i \tau - i \sigma _ { k } ) \left\lceil\left(\frac{1}{2}-i \tau-i \sigma_{k}\right)\right.\right. \\
\cdot \frac{M\left(x, x_{2} ; i \sigma_{k}\right)}{\left(\partial / \partial \sigma_{k}\right) M\left(x_{2}, x_{1} ; i \sigma_{k}\right)} F\left(\sigma_{k}\right) .
\end{array}
$$

Thus, at the points of continuity of $f(x)$ we obtain (2.1). We note that (2.1) becomes a result in [5] when $x_{1}=-1$ and $x_{2}=1$.

It follows from the foregoing theorem that, at points of continuity of $f(x)$, we have

$$
\begin{aligned}
f(x) & =\sum_{k} \sigma_{k}\left\lceil\left(\frac{1}{2}+i \tau-i \sigma_{k}\right) \Gamma\left(\frac{1}{2}-i \tau-i \sigma_{k}\right) \frac{R\left(x, x_{2} ; i \sigma_{k}\right)}{\left(\partial^{2} / \partial x_{2} \partial \sigma_{k}\right) R\left(x_{2}, x_{1} ; i \sigma_{k}\right)} F\left(\sigma_{k}\right)\right. \\
& +\frac{1}{2 \pi i} \int_{-\infty}^{\infty} \sigma\left\lceil\left(\frac{1}{2}+i \tau-i \sigma\right) \Gamma\left(\frac{1}{2}-i \tau-i \sigma\right) \frac{R\left(x, x_{2} ; i \sigma\right)}{\left(\partial / \partial x_{2}\right) R\left(x_{2}, x_{1} ; i \sigma\right)} F(\sigma) d \sigma\right.
\end{aligned}
$$

where

$$
\begin{gathered}
F(\sigma)=\int_{x_{1}}^{x_{2}} \frac{f(x)}{1-x^{2}} R\left(x, x_{1} ; i \sigma\right) d x,-1<x_{1}<x_{2}<1, \\
R(x, y ; i \sigma)=P_{-1 / 2+i \tau}^{i \sigma}(x) \frac{\partial}{\partial y} P_{-1 / 2+i \tau}^{i \sigma}(-y)-P_{-1 / 2+i \tau}^{i \sigma}(-x) \frac{\partial}{\partial y} P_{-1 / 2+i \tau}^{i \sigma}(y)
\end{gathered}
$$

and $\sigma_{k}{ }^{\prime} s, \sigma, \tau$ are real.

The integrand in (2.21) has singularities at $\sigma=\sigma_{k}(k$ is positive integers) which are simple poles along the positive $\sigma$-axis, where

$$
\frac{\partial}{\partial x_{2}} R\left(x, x_{1} ; i \sigma_{k}\right)=0,\left(\sigma_{k}>0\right) .
$$

To prove (2.21) we use the following asymptotic formulas for large $\mu$ :

$$
\begin{gathered}
\frac{\partial}{\partial x} P_{-1 / 2+i \tau}^{-\mu}(x)=-\frac{\mu}{\Gamma(1+\mu)} \frac{1}{(1-x)(1+x)}\left(\frac{1+x}{1-x}\right)^{-\mu / 2}\left[1+O\left(|\mu|^{-1}\right)\right], \\
\frac{\partial}{\partial x} P_{-1 / 2+i \tau}^{-\mu}(-x)=-\frac{\mu}{\Gamma(1+\mu)} \frac{1}{(1+x)(1-x)}\left(\frac{1-x}{1+x}\right)^{-\mu / 2}\left[1+O\left(|\mu|^{-1}\right)\right],
\end{gathered}
$$


The proof of (2.21) is similar to the proof in the section 2, and we do not reproduce it. We note that (2.21) becomes a result in [5] when $x_{1}=-1$ and $x_{2}=1$.

3. EXAMPLES.

We now give examples of expansions of some functions.

(1) $\left(1-x^{2}\right)^{\nu / 2}=\sum_{k} \sigma_{k}\left\lceil\left(\frac{1}{2}+i \tau-i \sigma_{k}\right)\left\lceil\left(\frac{1}{2}-i \tau-i \sigma_{k}\right) \frac{M\left(x, x_{2} ; i \sigma_{k}\right)}{\left(\partial / \partial \sigma_{k}\right) M\left(x_{2}, x_{1} ; i \sigma_{k}\right)}\right.\right.$

$$
\begin{array}{r}
\cdot \frac{2^{\nu}\lceil(1+\nu)}{\left(\nu^{2}+\sigma_{k}^{2}\right)}\left(\nu+i \sigma_{k}\right)\left[P_{\nu}^{-\nu}\left(x_{1}\right) M_{1}\left(x_{1}, x_{1} ; i \sigma_{k}\right)-P_{\nu}^{-\nu}\left(x_{2}\right) M_{1}\left(x_{2}, x_{1} ; i \sigma_{k}\right)\right] \\
+\frac{2^{\nu}\lceil(1+\nu)}{2 \pi i} \int_{-\infty}^{\infty} \frac{\sigma(\nu+i \sigma)}{\nu^{2}+\sigma^{2}}\left\lceil\left(\frac{1}{2}+i \tau-i \sigma\right) \Gamma\left(\frac{1}{2}-i \tau-i \sigma\right) \frac{M\left(x, x_{2} ; i \sigma\right)}{M\left(x_{2}, x_{1} ; i \sigma\right)}\right. \\
\cdot\left[P_{\nu}^{-\nu}\left(x_{1}\right) M_{1}\left(x_{1}, x_{1} ; i \sigma\right)-P_{\nu}^{-\nu}\left(x_{2}\right) M_{1}\left(x_{2}, x_{1} ; i \sigma\right)\right] d \sigma,
\end{array}
$$

where

$$
\left(-1<x_{1}<x<x_{2}<1\right)
$$

$$
\begin{gathered}
M(x, y ; i \sigma)=P_{\nu}^{i \sigma}(x) P_{\nu}^{i \sigma}(-y)-P_{\nu}^{i \sigma}(-x) P_{\nu}^{i \sigma}(y) \\
M_{1}(x, y ; i \sigma)=P_{\nu-1}^{i \sigma}(x) P_{\nu}^{i \sigma}(-y)-P_{\nu-1}^{i \sigma}(-x) P_{\nu}^{i \sigma}(y) \text { and } \nu=-1 / 2+i \tau
\end{gathered}
$$

(2)

$$
\begin{aligned}
& P_{\nu}^{\mu}(x)= \sum_{k} \sigma_{k}\left\lceil\left(\frac{1}{2}+i \tau-i \sigma_{k}\right) \Gamma\left(\frac{1}{2}-i \tau-i \sigma_{k}\right) \frac{M\left(x, x_{2} ; i \sigma_{k}\right)}{\left(\partial / \partial \sigma_{k}\right) M\left(x_{2}, x_{1} ; i \sigma_{k}\right)} \frac{1}{\left(\mu^{2}+\sigma_{k}^{2}\right)}\right. \\
& \cdot\left[(\nu+\mu) P_{\nu-1}^{\mu}\left(x_{2}\right) M\left(x_{2}, x_{1} ; i \sigma_{k}\right)+\left(\nu+i \sigma_{k}\right)\left\{P_{\nu}^{\mu}\left(x_{1}\right) M_{1}\left(x_{1}, x_{2} ; i \sigma_{k}\right)\right.\right. \\
&\left.\left.-P_{\nu}^{\mu}\left(x_{2}\right) M_{1}\left(x_{2}, x_{2} ; i \sigma_{k}\right)\right\}\right]+\frac{1}{2 \pi i} \int_{-\infty}^{\infty} \frac{\sigma}{\mu^{2}+\sigma^{2}}\left\lceil\left(\frac{1}{2}+i \tau-i \sigma\right) \Gamma\left(\frac{1}{2}-i \tau-i \sigma_{1}\right)\right. \\
& \cdot \frac{M\left(x, x_{2} ; i \sigma\right)}{M\left(x_{2}, x_{1} ; i \sigma\right)}\left[(\nu+\mu) P_{\nu-1}^{\mu}\left(x_{2}\right) M\left(x_{2}, x_{1} ; i \sigma\right)+(\nu+i \sigma)\left\{P_{\nu}^{\mu}\left(x_{1}\right)\right.\right. \\
&\left.\left.\cdot M_{1}\left(x_{1}, x_{2} ; i \sigma\right)-P_{\nu}^{\mu}\left(x_{2}\right) M_{1}\left(x_{2}, x_{1} ; i \sigma\right)\right\}\right] d \sigma
\end{aligned}
$$

In all these results the conditions under which the expansion theorem hold are satisfied.

ACKNOWLEDGEMENT. This research is supported by CSIR, New Delhi, through a research project No. 25(41)/EMR-II/88 administered by the Calcutta Mathematical Society.

\section{REFERENCES}

1. ERDÉLYI, A.; MAGNUS, W.; OBERHITTINGER, F. \& TRICOMI, F.G., Higher Transcendental Functions, Vol. 1, McGraw Hill Co., 1953.

2. FELSEN, L.B., Some new transform theorems involving Legendre functions, J. Math. Phys. 37 (1958), 188-191.

3. LEBEDEV, N.N. \& SKAL'SKAYA, I.P., Integral expansion of an arbitrary function in terms of spherical functions, PMM 30 (1966), 252-258.

4. LEBEDEV, N.N. \& SKAL'SKAYA, I.P., Expansion of an arbitrary function into an integral in terms of associated spherical functions, PMM 32 (1968), 421-427.

5. LEBEDEV, N.N. \& SKAL'SKAYA, I.P., Integral representations related to Mehler-Fok transformations, Differential Equations 22 (1986), 1050-1056.

6. MANDAL, B.N., An integral transform associated with degree of Legendre functions, Bull. Cal. Math. Soc. 63 (1971), 1-6. 
7. MANDAL, B.N., Note on an integral transform, Bull. Math. de la Soc. Math. de la R.S. de Roumanie 63 (1971), 87-93.

8. MANDAL, B.N. \& GUHA ROY, P., On a Mehler-Fok type integral transform, Appl. Math. Lett. 4 (1991), 29-32.

9. MANDAL, N. \& MANDAL, B.N., Integral representation of a function in terms of associated Legendre functions, Bull. Math. de la Soc. Math. de la R.S. de Roumanie 4 (1991), to appear.

10. SNEDDON, I.N., The Use of Integral Transforms, McGraw Hill Co., 1972. 


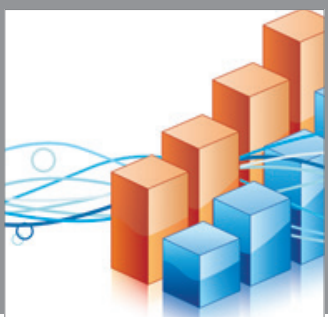

Advances in

Operations Research

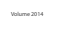

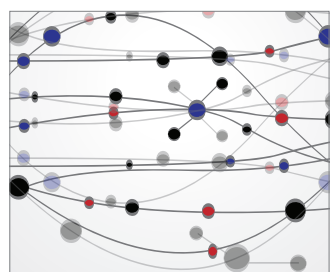

\section{The Scientific} World Journal
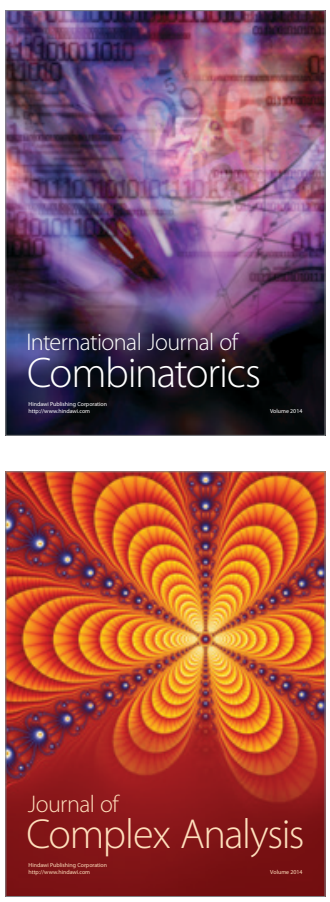

International Journal of

Mathematics and

Mathematical

Sciences
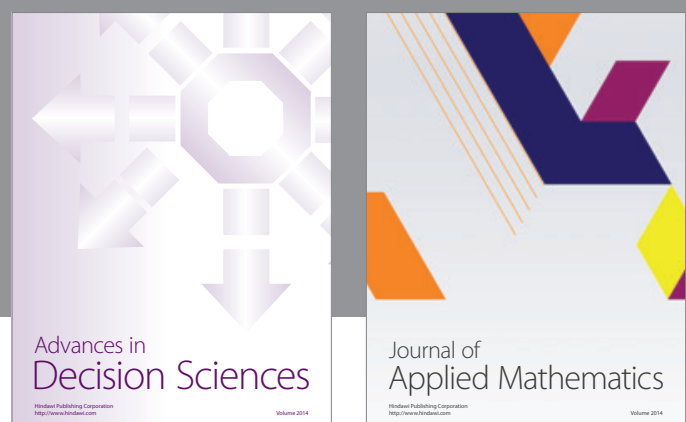

Journal of

Applied Mathematics
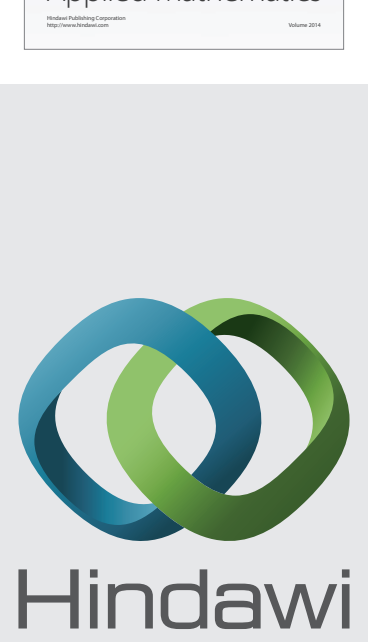

Submit your manuscripts at http://www.hindawi.com
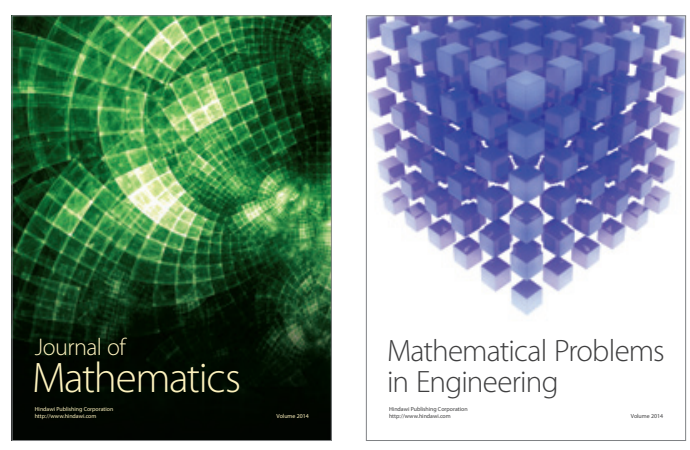

Mathematical Problems in Engineering
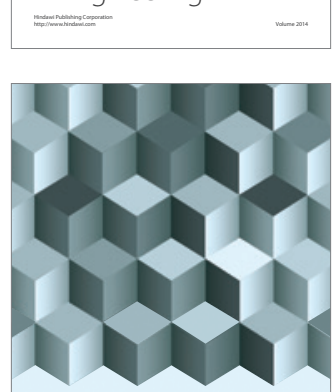

Journal of

Function Spaces
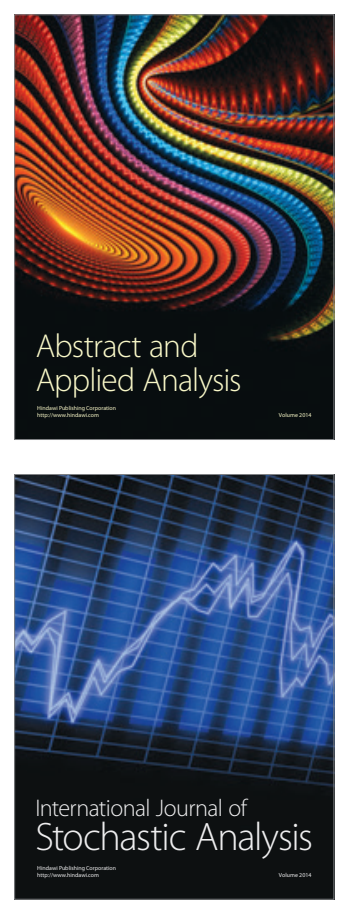

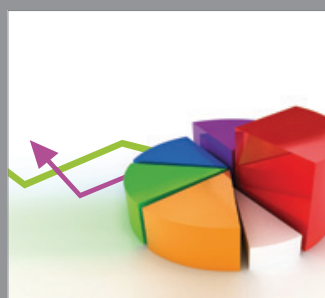

ournal of

Probability and Statistics

Promensencen
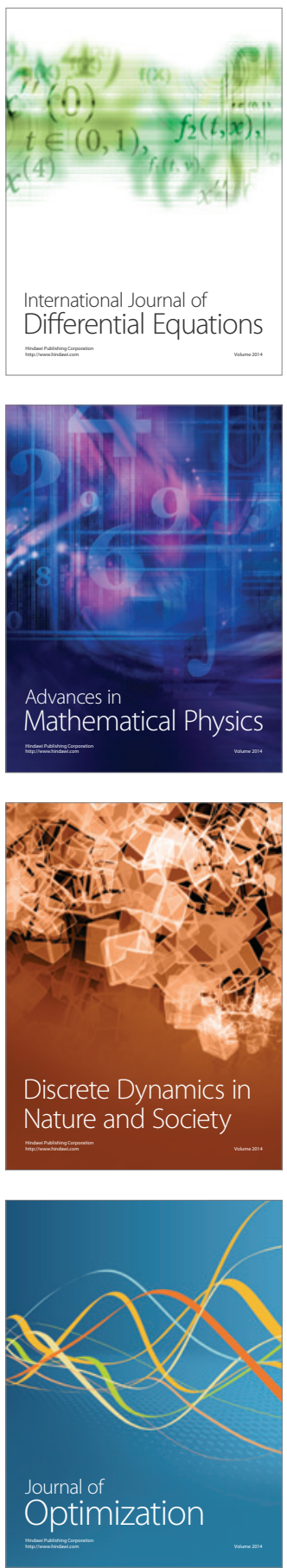